BMJ Open

Sport \&

Exercise

Medicine

\title{
Recruiting adult participants to physical activity intervention studies using sport: a systematic review
}

Rachel Cooke, Andy Jones

To cite: Cooke $R$, Jones $A$. Recruiting adult participants to physical activity intervention studies using sport: a systematic review. BMJ Open Sport Exerc Med 2017;3:e000231.

doi:10.1136/bmjsem-2017000231

- Additional material is published online only. To view please visit the journal online (http://dx.doi.org/ 10.1136/bmjsem-2017000231).

Accepted 9 April 2017

CrossMark

Norwich Medical School, University of East Anglia, Norwich, UK

Correspondence to Dr Rachel Cooke; rachel.I. cooke@uea.ac.uk

\section{ABSTRACT}

Objective To undertake a systematic review of the effectiveness of recruitment mechanisms for engaging and retaining target participants in sports interventions to promote physical activity behaviour change in adults.

Design A narrative systematic review of published studies providing details of the effectiveness of recruitment techniques used in interventions aimed at increasing physical activity via sport in adults.

Data sources Searches were conducted using five electronic databases, clinical trial registers, grey literature and snowballing from reference lists. All papers published in the English language were considered. The search was completed in November 2015.

Eligibility criteria All articles providing information on the recruitment of adults into interventions involving sport and reporting physical activity or participation outcomes were included.

Results Twenty-three studies met the inclusion criteria. The quality of recruitment reporting across included studies was generally classified as poor, lacking detailed descriptions of recruitment processes and providing insufficient reporting of recruitment outcomes. There was a distinct recruitment bias for more affluent, white, middle-aged women. Active-only recruitment techniques appeared to achieve a participant sample with more representative demographic characteristics than passive approaches. Conclusions Due to inadequate reporting and evaluation, the mechanisms for achieving effective recruitment and engagement in sport, particularly in hard-to-reach groups, are still unclear. Independent of recruitment mode, creating an intervention and context that reflect the interests and motivations of the target audience presents a promising area. There is an urgent need for more robust evaluation design and reporting of sports interventions.

\section{INTRODUCTION}

Physical inactivity is a global public health problem and the fourth leading cause of global mortality, resulting in over 5.3 million deaths a year worldwide. ${ }^{1}$ It costs $£ 0.9$ billion to the National Health Service in the UK alone. ${ }^{2}$ Sport presents a possible means of promoting physical

\section{What are the new findings?}

Evaluation and reporting of recruitment processes in sports interventions is scarce resulting in a lack of evidence for suitable engagement mechanisms, particularly in hard-toreach groups.

Active-only recruitment approaches achieved more representative samples for their target population than passive or combined techniques. It is of concern, however, that these approaches may be more vulnerable to limited participant engagement, thus requiring additional components such as motivational interviewing to encourage participation.

How might it impact on clinical practice in the near future?

For future sporting interventions, techniques involving active recruitment may be particularly appropriate to recruit hard-to-reach participants or to better achieve target participant demographic composition.

activity (PA) and health. However, little is known about how best to engage inactive individuals in sport to increase PA.

It is recognised that PA and sports participation are unequally distributed across society with gender, age, disability, education and socioeconomic status (SES) as determinants. ${ }^{4}$ Certain subgroups of the population are therefore more likely to be inactive and stand to gain significant health benefits from increasing their PA. ${ }^{5}$ These groups are frequently considered 'hard to reach', and as such, it is important to understand how they may be successfully engaged in health-promoting interventions. ${ }^{3}$ Nevertheless, historically there has been a significant recruitment bias in PA interventions that have predominantly recruited white, middle-class, middle-aged women, 
resulting in under-represented male, socioeconomically disadvantaged and minority ethnic populations. ${ }^{6}$ In light of this, sports interventions are becoming increasingly targeted in attempts to reduce health inequalities and engage underserved populations.

In general, academic journals have prioritised the publication of intervention findings above the evaluation and reporting of recruitment processes and outcomes. ${ }^{8} 9$ This is limiting because, independent of intervention efficacy, the viability of a programme will be determined by its ability to recruit sufficient numbers of eligible participants. ${ }^{10}$ This limitation is further exacerbated by the fact that there has been a general lack of evaluation of sports programmes, thus limiting the evidence base on how to engage often hard-to-reach inactive populations in sport. ${ }^{3} 11 \mathrm{We}$ argue that a better understanding of recruitment procedures and their effectiveness is needed to inform those wishing to successfully replicate or adapt interventions, particularly when informing policy or practice. $^{12}$ This review has therefore been undertaken to provide evidence for the role of planning, implementation and reporting of recruitment processes for sports interventions promoting positive PA behaviour change.

\section{METHOD}

This review systematically identifies and evaluates the effectiveness of recruitment techniques used in interventions aimed at increasing PA using sport. Although the definition of recruitment varies between studies, we have defined 'recruitment' to be those who enrol on an intervention independent of whether they participate. We further define recruitment effectiveness as engaging sufficient numbers of target populations to (1) register for the intervention, (2) participate, (3) complete the intervention or any follow-up, and (4) achieve long-term positive PA behaviour change. Consequently, for the purpose of this review, retention is also considered a component of recruitment effectiveness. From a methodological perspective, the two primary challenges were the identification of reports on PA interventions using sport and the subsequent identification of the recruitment methods used. Hence, the adopted methodology was designed to particularly address these issues.

Inclusion criteria and analysis methodology were previously specified and documented in a protocol registered as CRD42015015815 (available at http:// www.crd.york.ac.uk/PROSPERO/display_record.asp? ID $=$ CRD42015015815)

\section{Data sources}

To identify potential studies, searches were conducted using electronic databases, clinical trials registers, grey literature and snowballing from reference lists. This involved a systematic search of the following electronic databases: CINAHL, MEDLINE, EMBASE, PsycINFO and SPORTDiscus. Then the two primary organisations in England contributing to the delivery of sports programmes for PA and health (Sport England, the national body for sport in England, and UKActive, a not-for-profit health body for the PA sector in the UK) were contacted to identify additional grey literature. Titles and abstracts of identified material were checked against inclusion and exclusion criteria for suitability. Full articles were then acquired and assessed for inclusion. Snowballing was employed whereby, following inclusion, the reference lists of papers were searched and further articles considered for inclusion. The search was completed in November 2015.

The search terms used were developed using those of earlier systematic reviews on the evaluation of participation outcomes of sporting interventions ${ }^{13}$ and recruitment into walking interventions ${ }^{10}$ combined with an extensive list of sporting activities recognised by Sport England. ${ }^{14}$ Details of the search syntax used for electronic databases are provided in the online supplementary appendix. For clinical trials registers, 'sport' was the only search term within the title, using interventional studies in adults or seniors as limiters. The details of the full inclusion and exclusion criteria are provided in online supplementary table 1. Pragmatic considerations meant the search was limited to papers published in English. All programmes recruiting adults into interventions involving sport and reporting PA or participation outcomes were included.

\section{Study selection}

The first reviewer conducted a review of all identified studies to exclude duplicates and studies that clearly did not meet the inclusion criteria, for example, interventions looking at elite sport performance-related outcomes. To check the inclusion process, $15 \%$ of review articles were randomly selected at the abstract screening stage and screened by the second reviewer, and all papers were found to have been correctly excluded. Where duplicate studies presented, the journal article reporting the most recruitment data was analysed and other articles excluded unless multiple papers were found to report on distinctly different aspects of the intervention and still meet the inclusion criteria.

\section{Data extraction}

A data extraction table was developed by both authors guided by the protocols of other reviews looking at recruitment into health interventions for guidance. ${ }^{15-}$ 17 The resultant table summarised the characteristics of the study, population, sport intervention, recruitment, retention and outcomes. Corresponding data were then extracted from all included papers by the first and second reviewers and were transcribed into the table using Microsoft Excel. 


\section{Data synthesis}

We anticipated considerable heterogeneity between identified interventions and their recruitment approaches and therefore planned to employ a narrative synthesis of results. Extracted information was also used to synthesise additional data relating to the study quality, efficiency and effectiveness following the methodology of a similar review. ${ }^{10}$

\section{Assessment of quality of recruitment reporting}

Due to the specific focus of this review, we did not attempt to assess the general quality of studies but rather focused on the quality of reporting of recruitment. This was assessed using criteria previously developed by Foster $e t a l^{10}$ in their review of recruitment into walking intervention studies. Two reviewers independently assessed the quality of recruitment reporting in the studies regarding where the population was recruited, who conducted the recruitment, the time spent planning and preparing the recruitment, the time spent conducting the recruitment and the target population to be recruited. Each criterion was given a value of zero (absent or inadequately described) or one (explicitly described and present). Using the nomenclature of Foster $\mathrm{et} \mathrm{al},{ }^{10}$ studies scoring three or below overall were considered 'low quality' while those that scored between four and five were considered 'high quality'.

\section{Assessment of efficiency}

We also adopted the methodology of Foster $e t a l^{10}$ to evaluate the efficiency of recruitment processes within included studies. This involved calculating the recruitment rates and efficiency ratios, where possible, for each included study. The following values were sought: the total number of potential participants who could be eligible for study ('pool'), potential participants invited to participate in the study ('invited'), potential participants who responded to the invitation ('responded') and participants who were assessed as eligible to

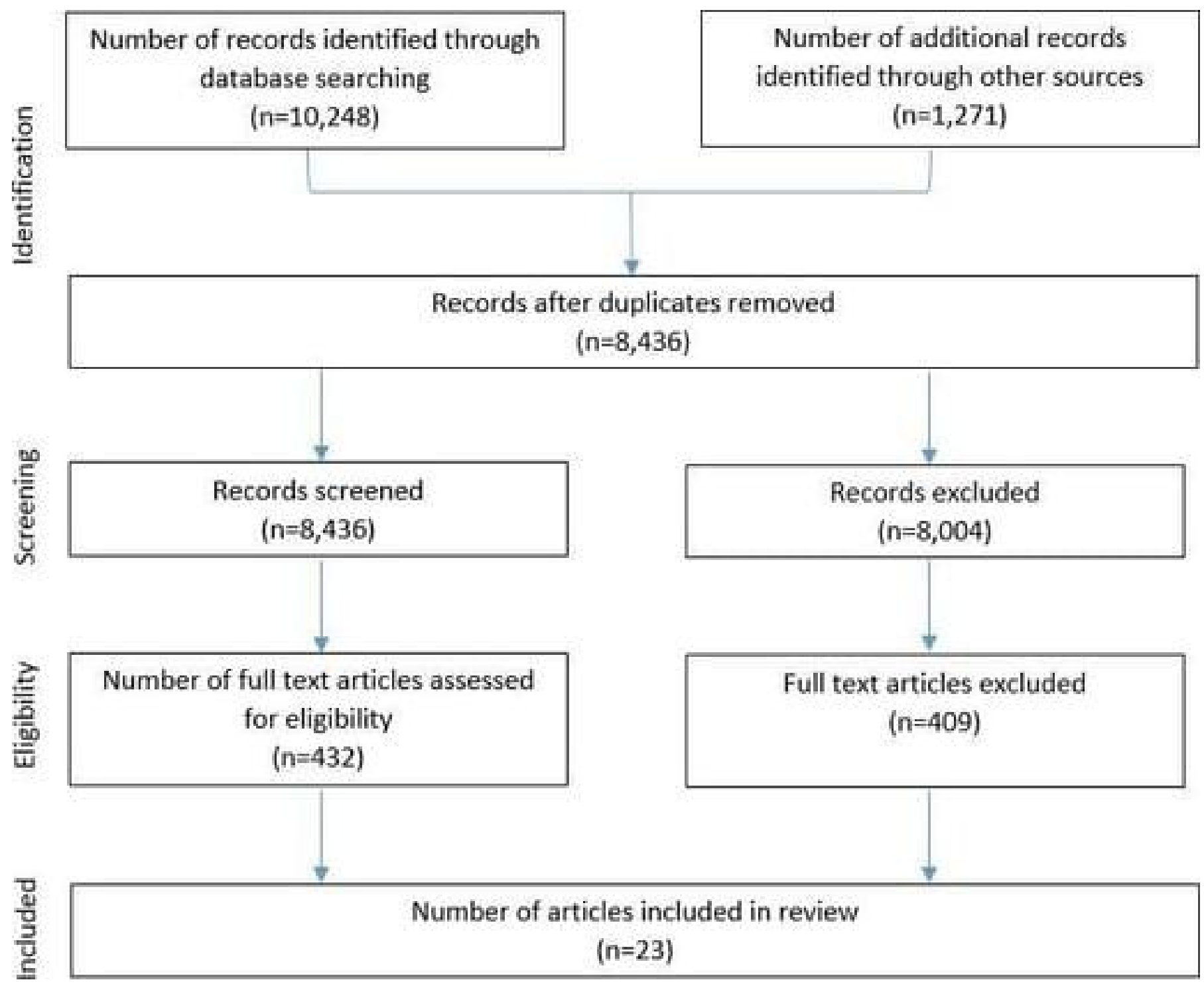

Figure 1 Review flow chart. 
participate and began the programme ('started'). Where possible, ratios were calculated for each stage, for example, by dividing the number of participants who 'started' the study by the total 'invited' the proportion taking up the intervention. Furthermore, a weekly rate of recruitment was calculated for those studies also providing recruitment duration.

\section{RESULTS}

\section{Study characteristics}

Twenty-three papers representing 22 interventions met our inclusion criteria. Figure 1 reports the flow of studies through the review process. Characteristics of included studies are presented in online supplementary table 2, ranked by quality score. Each included paper is referenced in the results and discussion sections in superscript using their reference citation. Full references for included papers are therefore listed in the bibliography. Studies were located in the UK $(\mathrm{n}=10),{ }^{18-27}$ USA $(\mathrm{n}=5),{ }^{28-32}$ Canada $(\mathrm{n}=3),{ }^{33-35}$ Norway $(n=2),{ }^{36} 37$ Italy $(n=1),{ }^{38}$ Switzerland $(n=1)^{39}$ and South America $(\mathrm{n}=1) \cdot{ }^{40}$ Nearly all the studies were quantitative experimental studies in design, with 9 randomised controlled trials, ${ }^{18}$ 27-30 323335402 nonrandomised controlled trials ${ }^{25} 37$ and 11 before-andafter studies. ${ }^{19} 21-24263134363839$ Of these, four were mixed methods and incorporated some qualitative design. ${ }^{19} 2128{ }^{34}$ We found only one qualitative study reporting on recruitment approaches. ${ }^{20}$

\section{Intervention characteristics}

There were a wide variety of intervention designs and sports demonstrated across the included studies (see online supplementary table 3). Twelve interventions involved multiple physical activities, all or some of which involved participating in sport, ${ }^{18-27} 36$ 37 while 11 offered an intervention using only one sporting activity. ${ }^{28-35} 38-40$ The main activities reportedly used, in isolation or combination, were dance $(\mathrm{n}=8),{ }^{23} 25293234373840$ football $(\mathrm{n}=7),{ }^{18-21} 232637$ exercise classes $(\mathrm{n}=7), \begin{array}{lllllll}19 & 24 & 25 & 27 & 31 & 37 & 38 \\ \text { running/ }\end{array}$ jogging $(n=6),{ }^{18} 2324333839$ swimming $(n=5)^{23} 2436-$ ${ }^{38}$ and yoga $(n=2) .{ }^{28} 30 \mathrm{~A}$ range of settings were used to deliver interventions, including professional football stadia and facilities, ${ }^{18-21}{ }^{26}$ leisure or sports facilities ${ }^{22}$ 2528313334 and non-sporting community sites such as schools and churches. ${ }^{27} 293638$ The average reported intervention duration was 23.7 weeks $(\mathrm{SD} \pm 27.7$ weeks, range 8-104 weeks).

\section{Characteristics of the participants}

Sample sizes (N started) of the studies ranged from 15 to 160018 participants. Twenty studies reported participant ages ${ }^{18-22} 2527-40$ with a mean age of 51.3 years (SD \pm 6.3 years) and range from 18 to 70 years (see online supplementary table 4). Six out of 22 studies that reported gender focused on recruiting female-only participants, ${ }^{24} 2932343539$ and five studies recruited only men. ${ }^{18} 20212633$ From the remaining 12 studies that did not recruit sex-specific groups, $67 \%$ (SD $\pm 16.2 \%$ ) of participants were women. Thirteen studies reported ethnicity. ${ }^{18-22} 25$ 29-35 Three studies reported targeting a single specific ethnic group: African-Americans, ${ }^{29}$ South Asians ${ }^{34}$ and 'coloured' (a term used by the authors) ethnicities. ${ }^{32}$ Of the remaining studies, 10 reported other ethnicity data; $80 \%$ of these participants were white Caucasian (SD $\pm 16 \%$, range 54\%-100\%). ${ }^{18-22} 2530313335$ Sociodemographic data (SES or income groups, education) were not consistently reported. An area-based index of multiple deprivation was reported in two studies, one of which reported the highest proportion of participants in the two least deprived quintiles $(5=25.1 \%$, $4=22.2 \%)^{18}$ and the other in the two most deprived quintiles $(1=25.7 \%, 2=20.29 \%) .{ }^{27}$ Average household income was below the poverty threshold in one study ${ }^{26}$ and indicative of a relatively high socioeconomic status in another. $^{32}$ Seven studies reported employment status. $^{20} 212830343638$ In six of the seven studies, the majority of participants were employed. ${ }^{20} 2128303436$

\section{Overview of recruitment reporting}

Five studies were classified as 'high' quality ${ }^{18} 28293334$ and the remaining 18 classified as 'low' quality in relation to recruitment reporting (see online supplementary table 5). All studies reported the setting where the recruitment of participants took place, but eight did not report who conducted the recruitment. $^{25-27} 32 \quad 36-38 \quad 40$ None of the included studies reported the time spent planning or preparing their recruitment, although six studies reported the time spent conducting recruitment. ${ }^{18} 2528293334$ All studies detailed the target population for recruitment (see online supplementary table 8).

\section{Recruitment data reported}

Because none of the studies reported the time spent planning or preparing the recruitment, no studies covered all stages of the recruitment process. All of the studies reported a specific target group and some details of where recruitment was conducted, although these were often non-specific (see online supplementary tables 6 and 8). Most popular were community settings $(n=14)$ such as community centres, ${ }^{31} 34$ sports clubs, ${ }^{18-21} 26$ places of worship $^{29}$ and locally distributed advertising/media. ${ }^{27}$ ${ }^{30}$ Medical or care settings were also popular $(n=6){ }^{24}$ 2530363840 Universities ${ }^{22} 35$ and workplaces were also used. $^{33} 39$

Seventeen studies reported who conducted the study recruitment. Most frequently reported recruiters were research staff $(n=5)$, which reflects the evaluative nature of much of the literature. ${ }^{18} 282935 \quad 39$ Seven studies reported the time spent on implementing recruitment, ${ }^{18} 252829333437$ which averaged as 52 
weeks (SD \pm 71 weeks, range 4-156 weeks) (see online supplementary table 6).

\section{Recruitment planning and implementation}

The reporting of recruitment methods was inconsistent and varied across studies (see online supplementary tables 6 and 7). The exact number of recruitment methods used was generally not disclosed and difficult to infer from the recruitment description. Five studies relied on one method of recruitment only, ${ }^{26} 283036$ 38 while 14 studies used two or more approaches. ${ }^{18} 21$ 23-25 27-29 31-33 353740 Recruitment approaches were categorised as 'passive' or 'active'. 'Passive' recruitment techniques prompt potential participants to identify themselves for the programme, ${ }^{41}$ whereas 'active' techniques require those involved in the programme to initiate contact with a potential participant (eg, health professional referrals). ${ }^{42}$ No relationship between the quality of recruitment reporting and the number of recruitment strategies used was observed. We did observe that while a number of studies used only passive techniques $(n=6),{ }^{27} 2830353940$ most used a mixture of active and passive $(\mathrm{n}=13)^{18-20} 22-252932-34$ 37 and a small number used only active methods $(n=3)$ (see online supplementary table 7). ${ }^{31} 3638$

\section{Recruitment rates and efficiencies}

We were unable to extract all of the values for the 'pool, invited, responded and started' participation levels required to calculate efficiencies across the recruitment process as set out by the Foster et $a l^{10}$ review (see online supplementary table 9). We were, however, able to calculate a weekly recruitment rate using the final number of participants divided by the time spent recruiting in weeks for six studies (mean 13 participants per week, $\mathrm{SD} \pm 14$, range $<1$ to 37 participants per week). Only one study reported the volume of participant uptake grouped by recruitment method. ${ }^{18}$

\section{Physical activity outcomes}

Physical activity outcomes were reported both directly, using validated measures of $\mathrm{PA}$, and indirectly through the reporting of attendance or participation across the included studies (see online supplementary table 10). Of those studies reporting change in PA, 14 reported significant increases in PA between baseline and the end of the intervention. ${ }^{18} 20212528293233 \quad 35-40$ Only five of these, however, reported maintaining a significant increase in PA from baseline at post-intervention follow-up ${ }^{32} 363^{38-40}$ with four reverting to non-significant differences. ${ }^{18} 2829 \quad 35$ Furthermore, one study reported a significant increase in PA at the post-intervention follow-up but not at the end of the intervention $^{30}$ and another ${ }^{27}$ had no significant changes in PA to report at the end of the intervention. In the studies that did not quantify PA, records of attendance were used to represent PA participation during the intervention period.

\section{Recruitment target, exclusion and study retention}

Sample size calculations were referenced in five studies, ${ }^{1827} 293637$ of which two indicated they were used to provide a target sample size for recruitment, ${ }^{18}$ ${ }^{29}$ which was successfully achieved by one ${ }^{18}$ and not the other. ${ }^{29}$ Fourteen studies indicated that there was a screening process to determine eligibility within the recruitment pathway. ${ }^{18} 2326$ 28-32 34-36 38-40 However, only five reported the proportion of recruits found to be ineligible at this stage (mean $18 \%, \mathrm{SD} \pm 17.9 \%){ }^{18} 28$ 293239 The number of recruited participants who did not attend the intervention was reported in six studies (mean 19\%, SD $\pm 22.9 \%$ ). ${ }^{26} 2931343540$ The cost of recruitment per participant was calculated in one study at $£ 20.32 .^{25}$ It was not clear in the majority of studies whether incentives for recruitment $(n=0)$ and retention $(n=2)^{1831}$ were offered. Attendance was reported in a variety of ways in 15 studies. ${ }^{18} 22$ 24-26 28 29 31-35 38-40 The average reported attendance was $77 \% \quad(\mathrm{n}=9$, $\mathrm{SD} \pm 12.4 \%$, range $57 \%-100 \%$ ).

Retention figures were reported in 16 studies. ${ }^{18} 1921$ 232526 29-31 33 35-40 Retention reporting could either refer to participation in follow-up (study retention) or participation in PA or sport (PA retention) as part of the intervention or beyond. The average reported study retention rate at first follow-up was $82 \%$ $\left(\mathrm{n}=13, \mathrm{SD} \pm 14.8\right.$, range 49-100), ${ }^{18} 1921262930333536$ 38-40 whereas the average reported PA retention rate was $28.5 \%(\mathrm{n}=2, \mathrm{SD} \pm 13.4$, range $19-38) .{ }^{23}{ }^{31}$ Number and reason for dropouts was reported in 11 studies. ${ }^{18}$ 25262930 33-36 3940 Most commonly cited reasons for dropout were illness or injury, work, unexpected commitments, lack of time, relocation or travel, and disliking the intervention.

\section{Additional comments reported regarding recruitment and retention}

Comments relating to recruitment highlighted word of mouth $^{192122} 2425$ and social media ${ }^{22-24}$ as valuable recruiters. Additionally, the role of recruitment partnerships ${ }^{19} 202324$ as well as active, ${ }^{24}$ passive $^{25}$ and multiple ${ }^{23-25}$ recruitment mechanisms were discussed. The design of promotional materials was highlighted in a number of studies. ${ }^{22-24}$ The intervention setting, ${ }^{20}$ ${ }^{21}$ appeal of the activity ${ }^{18} 19212225$ and opportunities for socialising ${ }^{19}{ }^{23}$ were also important for recruitment of the target group. Several studies commented on the successes $^{20} 21$ and challenges ${ }^{24} 27$ of reaching the target group.

Facilitators of retention discussed included social support, ${ }^{19} 212526343839$ variety of activities, ${ }^{19} 212224$ group cohesion, ${ }^{34} \quad 39 \quad 40$ fun/enjoyment, ${ }^{21} \quad 25 \quad 34$ coaching, ${ }^{24}{ }^{31}$ routine, $^{19}{ }^{21}$ accessibility of delivery site, ${ }^{21}$ competition, ${ }^{22}$ timing of sessions, ${ }^{24}$ 
affordability, ${ }^{24}$ use of incentives, ${ }^{24}$ availability of progression opportunities, ${ }^{24}$ high programme satisfaction $^{34}$ and higher baseline self-motivations towards PA. $^{39}$ Barriers to retention included dropout or nonattendance in the early stages of the intervention, ${ }^{18} 25$ if individual activity intervention rather than group was used $^{38}$ the appeal of the activity ${ }^{21}$ and degree of competition. $^{21}$

\section{DISCUSSION}

The effectiveness of any PA or sporting intervention is limited by the impact the intervention has on its participants and by the effectiveness of its recruitment of eligible participants to take part in the intervention. ${ }^{10}$ This systematic review showed that the evidence on how best to recruit is sparse due to the absence of generalisable findings and insufficient reporting of recruitment methodology and process outcomes in sports-based PA interventions. Furthermore, there is an absence of evidence linking specific recruitment methods to more successful long-term behaviour changes due to a lack of clarity surrounding recruitment channel outcomes. Lack of reported information meant we were also unable to asses the cost per person of recruitment for different recruitment strategies. This would be of particular interest as there is an inevitable cost-effect trade-off to be considered when designing recruitment mechanisms.

We know that reaching priority groups for PA studies, such as the inactive or unhealthy, socioeconomically disadvantaged, ethnic and other minority groups, is challenging. ${ }^{9}$ Our review demonstrates that current recruitment strategies engage predominantly white, middle-class, middle-aged women unless they are clearly designed to target specific demographic characteristics, such as gender or ethnicity. Furthermore, this recruitment bias for particular populations is supported by an earlier review of wider PA interventions. ${ }^{6}$ Our review also found that despite targeting some of these demographic characteristics successfully, interventions tended to achieve unrepresentative levels for the remaining untargeted characteristics such as socioeconomic status. For example, Vahabi and $\mathrm{Damba}^{34}$ recruited predominantly Indian women in a South Asian Bollywood dancing intervention. However, most of these women were in full-time employment and had a university degree or higher, suggesting they were of a relatively high socioeconomic status. These findings indicate that typical recruitment techniques adopted by sports PA interventions are not reaching those most in need. Furthermore, targeting specific isolated characteristics may not be sufficient to alleviate recruitment bias towards particular demographic characteristics, thus limiting the generalisability of findings for policy and practice.

The use of active-only recruitment techniques ${ }^{31} 3638$ (whereby those involved in the study or programme make the first contact with a participant) appeared to achieve a participant sample with more representative demographic characteristics than passive approaches (ie, prompt potential participants to identify themselves) not targeting specific demographics. This finding is supported by Mutrie et al's paper, ${ }^{9}$ which found that active techniques were less likely to encourage self-selection and introduce recruitment bias than passive techniques. For example, a recruitment and intervention partnership targeting low-income demographics via community health services (active) ${ }^{31}$ successfully represented at-risk populations $(52 \%$ women, $54 \%$ white, average income less than $100 \%$ of the federal poverty level). However, this study had a $63 \%$ non-use attrition rate, thus demonstrating a low conversion to participation. This supports existing evidence that place-based strategies (ie, recruiting participants from a location where they already aggregate) result in more representative samples, but a lower overall participation rate. ${ }^{9} 43$ There is, however, evidence from the Mangeri et al paper $^{38}$ that techniques such as motivational interviewing and offering group intervention activities may encourage higher levels of participation in actively recruited participants. These observations highlight the difficulties and tensions faced by sporting interventions for reaching those most at risk or need. Due to insufficient monitoring and evaluation of recruitment processes, we were unable to determine which specific methods were more effective at engaging particular populations.

\section{Setting}

In addition to recruitment mode, the reach of a programme is determined by the appeal of characteristics of the intervention to target participants. Further, research processes such as having to complete questionnaires or wear an accelerometer may also influence uptake and impact recruitment to the programme in general. This review highlights the diversity and effectiveness of a subgroup of interventions delivered in professional football (soccer) clubs for engaging target populations in PA and health-promoting activities. ${ }^{18-21}$ 26 The professional football context in which these programmes were delivered was reported as a powerful draw for participants. ${ }^{19-21}{ }^{44}$ Furthermore, the range of populations successfully targeted and recruited by these interventions demonstrates the wide reach of professional sports settings. Using football club facilities, events, branding and media channels were all thought to contribute to the engagement of target groups, extending beyond the fan base of the host club. ${ }^{45}$ These programmes, therefore, highlight the importance of delivering interventions in contexts that are appealing to their target group and that use preexisting interests and communities to achieve successful engagement. 


\section{Reporting}

The quality of recruitment reporting across included studies was generally classified as poor, with a distinct lack of information on how much time was spent planning and implementing recruitment. The majority of interventions reported using multiple, simultaneous recruitment methods, but only one reported the effectiveness of individual approaches. ${ }^{18}$ Instead, recruitment was largely generalised to a location or setting, thus making it unclear which method or combination of methods were most effective at recruiting particular target groups and achieving long-term behaviour change.

Higher level overviews of target populations were generally well reported. However, few studies described the pool of potential participants, the number of individuals invited to participate, how many responded and how many started the intervention, all of which were required to calculate recruitment efficiency in accordance with the Foster et al review. ${ }^{10}$ In fact, comparable levels of reporting were seen between studies included in our review and Foster et al's, ${ }^{10}$ thus restricting both reviews' ability to compute and compare efficiency ratios across included studies. Further to this, we also sought to investigate some process outcomes that are of interest when looking to translate research into practice: target sample size and the extent to which it was achieved, the eligibility and representativeness of recruited participants as well as their participation and retention in the intervention. Unfortunately, these were also poorly reported, meaning there is limited process-based evidence to inform recruitment designs that facilitate powered and representative sample sizes for rigorous evaluation.

The need for improved reporting highlighted in this review may in part be driven by a previous lack of agreement on a standard framework for recruitment reporting. In England, a standard evaluation framework (SEF) has recently been introduced to guide programmes to collect and evaluate information relating to PA interventions. ${ }^{12}$ The SEF advocates the monitoring and reporting of a range of recruitment variables as part of its essential criteria for evaluating PA interventions. These include method of recruitment, target population characteristics, measures of the flow of participants through the intervention and dates of crucial time points such as first point of contact and follow-ups. Following adequate execution and reporting of the essential recruitment information, such as those set out in the SEF, the future application of data extraction and scoring procedures attempted within this review would likely produce a greater insight into recruitment processes for sporting interventions aiming to promote $\mathrm{PA}$. We are unaware of similar guidance in other international settings, although these may exist. The level of measurement and reporting observed in this review, however, suggests that standard evaluation of recruitment variables is not being used elsewhere. In the UK, the SEF is a relatively recent document having been published in 2012, and as such its impact may yet to be seen in the academic literature. This may also be the case for other similar guidance, yet if comparable guidance has been in place for longer, our findings raise the question of the extent of its adoption and application.

\section{Strengths and limitations of this review}

The strength of this review is that it used protocols, previously shown to be successful in other reviews, to systematically identify and analyse 23 sport PA intervention papers using a comprehensive search strategy and extensive data extraction table. We also attempted to compute a set of metrics to describe recruitment efficiency that were informed by similar systematic reviews investigating sport or recruitment.

Regarding limitations, there are limited published or publicly available evaluations of sporting interventions published in English as well as a reluctance from editors to publish articles where recruitment is the prime focus. ${ }^{8}$ This restricted conclusions relating to a number of processes of interest for this review. In line with the objectives of this study, we assessed the quality of recruitment reporting as our risk of bias tool. An implication of this was that we did not assess the overall quality of the included study. The lack of rigorous recruitment monitoring and reporting meant that the quality of extracted information on recruitment was not enough to allow a meta-analysis to be taken. A further issue in this review was that a number of the programmes considered were designed as research studies and consequently include additional research processes otherwise absent in typical sports interventions. There is evidence that characteristics of the population recruited can be influenced by recruitment protocols, intervention and research design characteristics. $^{9}$ Research protocols may therefore impact recruitment differently to unevaluated sports programmes or those with a lighter-touch evaluation. Consequently, the generalisability of our results was limited by the difficulties of separating the effect of the research and evaluation from overall programme recruitment. Furthermore, extracted values for participant outcomes were limited to only those who participated in the reported evaluation and may not be representative of the complete participant population.

\section{CONCLUSIONS}

Overall, this review emphasises the need for robust evaluation design and reporting of sports intervention and recruitment processes to permit future evidencebased interventions. There is a growing evidence base for the benefits of sport for physical inactivity and health. However, due to inadequate reporting and evaluation, the mechanisms for achieving effective recruitment and engagement in sport, particularly in 
hard-to-reach groups, are still unclear. There is a notable tendency of sporting interventions to recruit white, more affluent, middle-aged women. Simply targeting isolated demographic characteristics, such as gender or ethnicity, appears insufficient to recruit a sample representative of the population for the remaining, untargeted characteristics. Combinations of active and passive methods were commonly used, yet active-only recruitment approaches achieved more representative samples for their target population. It is of concern, however, that active-only recruitment may be more vulnerable to limited participant engagement, thus requiring additional components such as motivational interviewing to encourage participation. Independent of recruitment mode, creating an intervention and context that reflect the interests and motivations of the target audience, such as local professional football facilities, presents a promising area.

Acknowledgements We would like to thank the authors for their correspondence and contribution to the collected data. We would also like to thank $\mathrm{Dr}$ Charlie Foster for his input and advice that helped inform the systematic review protocol, Ryan Love for acting as a second reviewer, William Jones, faculty librarian, for his help in the search strategy and Professor Marc Suhrcke, Professor Richard Fordham, Dr Victoria Warburton and Dr Lee Beaumont for their guidance and support. Funding from Sport England is gratefully acknowledged.

Contributors Charlie Foster gave input and advice that helped inform the systematic review protocol. Ryan Love acted as a second reviewer. William Jones, faculty librarian, helped with the search strategy. Professor Marc Suhrcke, Professor Richard Fordham, Dr Victoria Warburton and Dr Lee Beaumont provided guidance and support.

Funding Funding from Sport England is gratefully acknowledged. The work was undertaken under the auspices of the Centre for Diet and Activity Research (CEDAR), a UKCRC Public Health Research Centre of Excellence (RES-590-28-0002) that is funded by the British Heart Foundation, Cancer Research UK, Economic and Social Research Council, Medical Research Council, the National Institute for Health Research and the Wellcome Trust.

Competing interests None declared.

Provenance and peer review Not commissioned; externally peer reviewed.

Open Access This is an Open Access article distributed in accordance with the terms of the Creative Commons Attribution (CC BY 4.0) license, which permits others to distribute, remix, adapt and build upon this work, for commercial use, provided the original work is properly cited. See: http:// creativecommons.org/licenses/by/4.0/

(c) Article author(s) (or their employer(s) unless otherwise stated in the text of the article) 2017. All rights reserved. No commercial use is permitted unless otherwise expressly granted.

\section{REFERENCES}

1. Lee IM, Shiroma EJ, Lobelo F, et al. Effect of physical inactivity on major non-communicable diseases worldwide: an analysis of burden of disease and life expectancy. Lancet 2012;380:219-29.

2. Scarborough $P$, Bhatnagar $P$, Wickramasinghe $K K$, et al. The economic burden of ill health due to diet, physical inactivity, smoking, alcohol and obesity in the UK: an update to 2006-07 NHS costs. J Public Health 2011;33:527-35.

3. Cavill N, Richardson D, Foster C. Sport England Inactivity Sport Report. 2012a. https://www.sportengland.org/media/103075/fullreport-inactivity-sport.pdf. (accessed 26 Oct 2015).

4. Roberts K, Cavill N, Hancock C, et al. Social and economic inequalities in diet and physical activity. 2013. http://www.noo.org.
uk/uploads/doc/vid_19253_Social_and_economic_inequalities_in diet_and_physical_activity_04.11.13.pdf. (accessed 17 Feb 2016).

5. Warburton DE, Nicol CW, Bredin SS. Health benefits of physical activity: the evidence. CMAJ 2006;174:801-9.

6. Waters LA, Galichet B, Owen N, et al. Who participates in physical activity intervention trials? J Phys Act Health 2011;8:85-103.

7. Fund BL. How do we engage harder to reach groups in physical activity? 2009. https://www.biglotteryfund.org.uk/-/media/Files/ Research\%20Documents/er_csi-eval_yr3.pdf. (accessed 17 Feb 2016).

8. Froelicher ES, Lorig K. Who cares about recruitment anyway? Patient Educ Couns 2002;48:97.

9. Mutrie N, Foster C, Estabrooks $\mathrm{P}$, et al. Recruiting hard-to-reach populations to physical activity studies: evidence and experiences. $J$ Phys Activity and Health 2010;7:329-31.

10. Foster CE, Brennan G, Matthews A, et al. Recruiting participants to walking intervention studies: a systematic review. Int J Behav Nutr Phys Act 2011;8:137.

11. Coalter F. A wider social role for sport: whos keeping the score? London: Taylor \& Francis, 2007.

12. Cavill N, Roberts K, Rutter H, et al. Standard evaluation framework for physical activity interventions. 2012b. http://www.noo.org.uk/ uploads/doc/vid_16722_SEF_PA.pdf. (accessed 4 Apr 2016).

13. Jackson N, Howes F, Gupta S, et al. Interventions implemented through sporting organisations for increasing participation in sport (review). The Cochrane Library 2005.

14. Sport England. Sports that we recognise. 2014a. https://www.sportengland.org/our-work/national-governing-bodies/sports-that-werecognise/. (accessed 9 Sep 2015).

15. Shorter G, Ferry F, Maguire L, et al. Recruitment and retention strategies in trials of online interventions designed to change alcohol use behaviour: protocol. PROSPERO, 2014. http://www.crd.york.ac. uk/PROSPERO/display_record.asp?ID=CRD42014009804. (accessed 17 Feb 2016).

16. Pijnenborg M, Vogelaar S, Van der Marck M, et al. Effective recruitment and selection interventions with elderly people in health related research: protocol. PROSPERO 2014, 2014. http://www.crd. york.ac.uk/PROSPERO/display_record.asp?ID=CRD42014009105.

17. Partridge S, Lam E, Allman-Farinelli M. Strategies for successful recruitment of young adults to healthy lifestyle programs for the prevention of weight gain: a systematic review: protocol. PROSPERO 2015, 2015. http://www.crd.york.ac.uk/PROSPERO/display_record. asp?ID=CRD42015017761. (accessed 17 Feb 2016).

18. Wyke S, Hunt K, Gray CM, et al. Football Fans in Training (FFIT): a randomised controlled trial of a gender-sensitised weight loss and healthy living programme for men ? end of study report. Public Health Res 2015;3:1-130.

19. Parnell D, Pringle A, McKenna J, et al. Reaching older people with PA delivered in football clubs: the reach, adoption and implementation characteristics of the Extra Time Programme. BMC Public Health 2015;15:220.

20. Pringle A, Zwolinsky S, McKenna J, et al. Effect of a national programme of men's health delivered in English Premier League football clubs. Public Health 2013;127:18-26.

21. Pringle A, Zwolinsky S, McKenna J, et al. Health improvement for men and hard-to-engage-men delivered in English Premier League football clubs. Health Educ Res 2014;29:503-20.

22. Sport England. Active Universities Final Report. 2014b. https://www. sportengland.org/media/1221038/active-universities-final-report.pdf. (accessed 8 Mar 2016).

23. Sport England. Get Healthy Get Active what weve Learnt so far. 2014c. https://www.sportengland.org/media/387892/Get-HealthyGet-Active-what-we-ve-learnt-FINAL.pdf. (accessed 8 Mar 2016).

24. Oldfield K, Vivian D. Evaluation of the active women Programme interim report prepared forsport England by IFF research. 2012 https://www.sportengland.org/media/39605/active-womenprogramme-first-year-evaluation-report.pdf (accessed 8 Mar 2016).

25. Withall J, Jago R, Fox KR. The effect a of community-based social marketing campaign on recruitment and retention of low-income groups into physical activity programmes-a controlled before-andafter study. BMC Public Health 2012;12:836.

26. Brady AJ, Perry C, Murdoch DL, et al. Sustained benefits of a health project for middle-aged football supporters, at Glasgow Celtic and Glasgow Rangers Football Clubs. Eur Heart J 2010;31:2966-8.

27. Solomon E, Rees T, Ukoumunne OC, et al. The Devon Active Villages Evaluation (DAVE) trial of a community-level physical activity intervention in rural south-west England: a stepped wedge cluster randomised controlled trial. Int J Behav Nutr Phys Act 2014;11:94. 
28. Bryan S, Pinto Zipp G, Parasher R. The effects of yoga on psychosocial variables and exercise adherence: a randomized, controlled pilot study. Altern Ther Health Med 2012;18:50-9.

29. Murrock C. Dance and physical activity in African American women. Department of Nursing, Case Western University 2007.

30. Alexander G, Innes KE, Bourguignon C, et al. Patterns of yoga practice and physical activity following a yoga intervention for adults with or at risk for type 2 diabetes. J Phys Act Health 2012;9:53-61.

31. Boyd ST, Scott DM, Augustine SC. Exercise for low-income patients with diabetes. Diabetes Educ 2006;32:385-93.

32. Lee RE, Mama SK, Medina A, et al. SALSA : SAving lives staying active to promote Physical activity and healthy eating. $J$ Obes 2011;2011:1-7.

33. Cunningham DA, Rechnitzer PA, Howard $\mathrm{JH}$, et al. Exercise training of men at retirement: a clinical trial. J Gerontol 1987;42:17-23.

34. Vahabi M, Damba C. A feasibility study of a culturally and genderspecific dance to promote physical activity for South Asian immigrant women in the greater Toronto area. Womens Health Issues 2015;25:79-87.

35. McDonough MH, Sabiston CM, Sedgwick WA, et al. Changes in intrinsic motivation and physical activity among overweight women in a 12-Week Dragon Boat Exercise intervention study. WSPAJ 2010;19:33-46.

36. Jepsen R, Aadland E, Robertson L, et al. Physical activity and quality of life in severely obese adults during a two-year lifestyle intervention programme. J Obes 2015;2015:1-11.

37. Lupton BS, Fønnebø V, Søgaard AJ. Finnmark Intervention Study. The Finnmark intervention study: is it possible to change CVD risk factors by community-based intervention in an Arctic village in crisis? Scand J Public Health 2003;31:178-86.
38. Mangeri F, Montesi L, Forlani G, et al. A standard ballroom and Latin dance program to improve fitness and adherence to physical activity in individuals with type 2 diabetes and in obesity. Diabetol Metab Syndr 2014;6:74.

39. Baschung Pfister P, Niedermann K, Sidelnikov E, et al. Active over 45: a step-up jogging programme for inactive female hospital staff members aged 45+. Eur J Public Health 2013;23:817-22.

40. Foster ER, Golden L, Duncan RP, et al. Community-based Argentine tango dance program is associated with increased activity participation among individuals with Parkinson's disease. Arch Phys Med Rehabil 2013;94:240-9.

41. Lee RE, McGinnis KA, Sallis JF, et al. Active vs. passive methods of recruiting ethnic minority women to a health promotion program. Ann Behav Med 1997:19:378-84.

42. Raynor HA, Osterholt KM, Hart CN, et al. Evaluation of active and passive recruitment methods used in randomized controlled trials targeting pediatric obesity. Int J Pediatr Obes 2009;4:224-32.

43. Almeida FA, Smith-Ray RL, Van Den Berg R, et al. Utilizing a simple stimulus control strategy to increase physician referrals for physical activity promotion. J Sport Exer Psych 2005;27:505-14.

44. Gray C, Hunt K, Mutrie N, et al. Can the draw of professional football clubs help promote weight loss in overweight and obese men? A feasibility study of the football fans in training programme delivered through the Scottish Premier League. J Epidemiol Commu 2011;65:A37-A38.

45. Curran $\mathrm{K}$, Bingham DD, Richardson D, et al. Ethnographic engagement from within a football in the community programme at an English Premier League football club. Soccer \& Society 2014;15:934-50. 